Centre interuniversitaire de recherche en économie quantitative

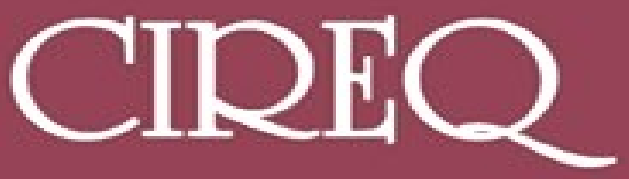

Cahier 27-2005

DOMAIN CLOSEDNESS CONDITIONS AND RATIONAL CHOICE

Walter BOSSERT and Kotaro SUZUMURA 


\section{CIREQ}

Le Centre interuniversitaire de recherche en économie quantitative (CIREQ) regroupe des chercheurs dans les domaines de l'économétrie, la théorie de la décision, la macroéconomie et marchés financiers, la microéconomie appliquée et économie expérimentale et l'économie de l'environnement et des ressources naturelles. Ils proviennent principalement des universités de Montréal, McGill et Concordia. Le CIREQ offre un milieu dynamique de recherche en économie quantitative grâce au grand nombre d'activités qu'il organise (séminaires, ateliers, colloques) et de collaborateurs qu'il reçoit chaque année.

The Center for Interuniversity Research in Quantitative Economics (CIREQ) regroups researchers in the fields of econometrics, decision theory, macroeconomics and financial markets, applied microeconomics and experimental economics, and environmental and natural resources economics. They come mainly from the Université de Montréal, McGill University and Concordia University. CIREQ offers a dynamic environment of research in quantitative economics thanks to the large number of activities that it organizes (seminars, workshops, conferences) and to the visitors it receives every year.

\section{Cahier 27-2005}

\section{DOMAIN CLOSEDNESS CONDITIONS AND RATIONAL CHOICE}

\section{Walter BOSSERT and Kotaro SUZUMURA}

téléphone : (514) 343-6557

télécopieur : (514) 343-5831 cireq@umontreal.ca http://www.cireq.umontreal.ca
Université th Concordia 
Ce cahier a également été publié par le Département de sciences économiques de l'Université de Montréal sous le numéro 2005-21.

This working paper was also published by the Department of Economics of the University of Montreal, under number 2005-21.

Dépôt légal, Bibliothèque nationale du Canada, 2005, ISSN 0821-4441

Dépôt légal, Bibliothèque nationale du Québec, 2005, ISBN 2-89382-521-4 


\title{
Domain Closedness Conditions and Rational Choice*
}

\author{
WALTER Bossert \\ Département de Sciences Economiques and CIREQ \\ Université de Montréal \\ C.P. 6128, succursale Centre-ville \\ Montréal QC H3C 3J7 \\ Canada \\ walter.bossert@umontreal.ca
}

Kotaro Suzumura

Institute of Economic Research

Hitotsubashi University

Kunitachi, Tokyo 186-8603

Japan

suzumura@ier.hit-u.ac.jp

This version: November 2005

* Financial support through grants from the Social Sciences and Humanities Research Council of Canada, the Fonds pour la Formation de Chercheurs et l'Aide à la Recherche of Québec and a Grant-in-Aid for Scientific Research from the Ministry of Education, Culture, Sports, Science and Technology of Japan is gratefully acknowledged. 


\section{Abstract}

The rationalizability of a choice function on an arbitrary domain under various coherence properties has received a considerable amount of attention both in the long-established and in the recent literature. Because domain closedness conditions play an important role in much of rational choice theory, we examine the consequences of these requirements on the logical relationships among different versions of rationalizability. It turns out that closedness under intersection does not lead to any results differing from those obtained on arbitrary domains. In contrast, closedness under union allows us to prove an additional implication. Journal of Economic Literature Classification No.: D11.

Keywords: Rational Choice, Domain Closedness, Coherence Properties. 


\section{Introduction}

A choice function selects a non-empty subset from each set of feasible alternatives within its domain. This choice function is rationalizable if there exists a binary relation on the universal set of alternatives such that these choices are performed in accordance with this relation. The two fundamental forms of rationalizability are greatest-element rationalizability (G-rationalizability, for short) and maximal-element rationalizability (also referred to as M-rationalizability). Grationalizability requires that each element of the set of chosen objects is at least as good as all elements of the feasible set according to this relation, to be called a G-rationalization. Mrationalizability demands that the chosen set consists of all elements that are not dominated by any feasible alternative. That is, according to the relation in question (to be called an $M$ rationalization), the set of chosen objects is the set of alternatives for which there exists no feasible alternative that is strictly preferred.

In addition to the basic notion of rationalizability, various richness properties or coherence properties may be imposed on a rationalization. We use the term richness condition for the properties of reflexivity and completeness, whereas coherence properties are given by transitivity, consistency, quasi-transitivity and P-acyclicity. Depending on the combination of the notion of rationalizability employed, the richness conditions required and the coherence condition imposed, various definitions of rationalizability can be obtained. The logical relationships among these definitions of rationality on arbitrary domains have been examined in Bossert, Sprumont and Suzumura (2005a; 2005b; 2005c) and they are summarized in Bossert and Suzumura (2005).

The rationalizability properties on arbitrary domains involving transitive rationalizations or rationalizations without any additional properties have been characterized by Richter (1966, 1971). In contrast, rationalizability on such arbitrary domains involving weaker coherence properties has received little attention until recently. Due to the contributions of Bossert, Sprumont and Suzumura (2005a; 2005b) and of Bossert and Suzumura (2005), characterizations of all notions of rationalizability are now available.

The generality involved in considering arbitrary domains is obtained at the cost of ignoring any structural properties the domain of a choice function may possess in specific contexts. An important class of domain assumptions that are plausible in many circumstances (see Section 3 for examples) is based on set-theoretic closedness properties. The two domain closedness properties that have received the most attention are closedness under union and closedness under intersection. Closedness under union requires that, for any collection of sets each of which belongs to the domain of a choice function, their union is a member of this domain as well. Closedness under intersection demands that, whenever the members of a collection of sets are in 
the domain of a choice function and their intersection is non-empty, then this intersection is an element of the domain as well.

Domain closedness conditions have been employed in the context of transitive rationalizability, where they are proven to be very useful in providing considerable simplifications of some results. The purpose of this paper is to examine whether these domain restrictions also allow us to simplify the logical relationships among the notions of rationalizability that can be identified. It turns out that this is not the case for closedness under intersection: this domain restriction is not sufficient to change the logical relationships among the rationalizability axioms that are obtained on arbitrary domains. On the other hand, if closedness under union is imposed, an additional implication emerges. The proof of this new result, which employs the axiom of choice, is of some interest in its own right. It establishes properties of more general sets than partially ordered sets.

Our basic definitions and some preliminary observations are presented in Section 2. Section 3 contains our analysis of the logical relationships between the various notions of rationalizability in the presence of domain closedness assumptions. Finally, Section 4 concludes.

\section{Preliminaries}

Let $X$ be a non-empty (but otherwise arbitrary) universal set of alternatives, and suppose $R \subseteq$ $X \times X$ is a (binary) relation on $X$. The asymmetric factor $P(R)$ of $R$ is defined by

$$
P(R)=\{(x, y) \in X \times X \mid(x, y) \in R \text { and }(y, x) \notin R\},
$$

and the symmetric factor $I(R)$ of $R$ is given by

$$
I(R)=\{(x, y) \in X \times X \mid(x, y) \in R \text { and }(y, x) \in R\} .
$$

We interpret $R$ as a weak preference relation. Accordingly, $P(R)$ and $I(R)$ are interpreted as the strict preference relation and the indifference relation corresponding to $R$, respectively. The diagonal relation on $X$ is given by $\Delta=\{(x, x) \mid x \in X\}$.

Let $\mathbb{N}$ denote the set of positive integers. The following properties of a binary relation $R$ are of importance in this paper.

Reflexivity. For all $x \in X$,

$$
(x, x) \in R
$$

Completeness. For all $x, y \in X$ such that $x \neq y$,

$$
(x, y) \in R \text { or }(y, x) \in R .
$$


Transitivity. For all $x, y, z \in X$,

$$
[(x, y) \in R \text { and }(y, z) \in R] \Rightarrow(x, z) \in R .
$$

Consistency. For all $K \in \mathbb{N} \backslash\{1\}$ and for all $x^{0}, \ldots, x^{K} \in X$,

$$
\left(x^{k-1}, x^{k}\right) \in R \text { for all } k \in\{1, \ldots, K\} \Rightarrow\left(x^{K}, x^{0}\right) \notin P(R) .
$$

Quasi-transitivity. For all $x, y, z \in X$,

$$
[(x, y) \in P(R) \text { and }(y, z) \in P(R)] \Rightarrow(x, z) \in P(R) .
$$

P-acyclicity. For all $K \in \mathbb{N} \backslash\{1\}$ and for all $x^{0}, \ldots, x^{K} \in X$,

$$
\left(x^{k-1}, x^{k}\right) \in P(R) \text { for all } k \in\{1, \ldots, K\} \Rightarrow\left(x^{K}, x^{0}\right) \notin P(R) .
$$

A reflexive and transitive relation is called a quasi-ordering and a complete quasi-ordering is called an ordering.

We refer to reflexivity and completeness as richness conditions. This term is motivated by the observation that the properties in this group require that, at least, some pairs must belong to the relation under consideration. In the case of reflexivity, all pairs of the form $(x, x)$ are required to be in the relation, whereas completeness demands that, for any two distinct alternatives $x$ and $y$, at least one of $(x, y)$ and $(y, x)$ must be in $R$. Clearly, the reflexivity requirement is equivalent to the set inclusion $\Delta \subseteq R$.

Transitivity, consistency, quasi-transitivity and P-acyclicity are what we call coherence properties. They require that if specific pairs belong to $R$, then certain other pairs must belong to $R$ as well (as is the case for transitivity and for quasi-transitivity) or certain other pairs must not belong to $R$ (which applies to consistency and to P-acyclicity). Quasi-transitivity and consistency are independent properties. Transitivity implies quasi-transitivity which, in turn, implies P-acyclicity. Moreover, transitivity implies consistency, and consistency implies P-acyclicity. The reverse implications are not true in general. However, the distinction between transitivity and consistency disappears for a reflexive and complete relation; see Suzumura (1983, p.244). Thus, if a relation $R$ on $X$ is reflexive, complete and consistent, then $R$ is transitive, hence an ordering.

Transitivity is the classical coherence requirement on preference relations and its significance in theories of individual and collective choice is evident. Quasi-transitivity was introduced by Sen $\left(1969 ; 1970\right.$, Chapter $\left.1^{*}\right)$, and it has been employed in numerous approaches to the theory 
of individual and social choice, including issues related to rationalizability. P-acyclicity has the important property that it, together with reflexivity and completeness, is not only sufficient for the existence of undominated choices from any arbitrary finite subset of a universal set, but it is also necessary for the existence of such choices from all possible finite subsets of the universal set; see Sen (1970, Chapter $\left.1^{*}\right)$.

Violations of transitivity are quite likely to be observed in practical choice situations. For instance, Luce's (1956) well-known coffee-sugar example provides a plausible argument against assuming that indifference is always transitive: the inability of a decision maker to perceive 'small' differences in alternatives is bound to lead to intransitivities. As this example illustrates, transitivity frequently is too strong an assumption to impose in the context of individual choice. In collective choice problems, it is even more evident that the plausibility of transitivity can be questioned. The concept of consistency, which is due to Suzumura (1976), is of particular interest in this context. As established by Suzumura (1976; 1983, Chapter 1), consistency is necessary and sufficient for the existence of an ordering which subsumes all the pairwise information contained in the binary relation. This result generalizes Szpilrajn's (1930) classical result on extending quasi-orderings to orderings. Suzumura's (1976) result was subsequently used by Donaldson and Weymark (1998) in their proof that every quasi-ordering is the intersection of a collection of orderings; this result extends Dushnik and Miller's (1941) fundamental observation on intersections of linear orders to the case where indifference is permitted. See Bossert (1999) for an alternative proof of Donaldson and Weymark's (1998) result that is based on Szpilrajn's (1930) theorem.

Let $\mathcal{X}$ be the set of all non-empty subsets of $X$, let $R$ be a relation on $X$ and suppose $S \in \mathcal{X}$. The set $G(S, R)$ of all $R$-greatest elements of $S$ is defined by

$$
G(S, R)=\{x \in S \mid(x, y) \in R \text { for all } y \in S\}
$$

and the set $M(S, R)$ of all $R$-maximal elements of $S$ is defined by

$$
M(S, R)=\{x \in S \mid(y, x) \notin P(R) \text { for all } y \in S\}
$$

As is straightforward to verify, $G(S, R)$ is contained in $M(S, R)$ for all relations $R$ on $X$ and for all $S \in \mathcal{X}$. Furthermore, if $R$ is reflexive and complete, then $G(S, R)=M(S, R)$; for relations $R$ that are not reflexive or not complete, the set inclusion can be strict.

A choice function is a mapping that assigns, to each feasible set in its domain, a subset of chosen alternatives from this feasible set. The domain of the choice function depends on the choice situation to be analyzed, but it will always be a set of subsets of $X$, that is, a subset of $\mathcal{X}$. We assume this subset of $\mathcal{X}$ to be non-empty to avoid degenerate situations. Thus, letting $\Sigma \subseteq \mathcal{X}$ 
be a non-empty domain, a choice function defined on that domain is a mapping $C: \Sigma \rightarrow \mathcal{X}$ such that, for all $S \in \Sigma, C(S) \subseteq S$. The image of $\Sigma$ under $C$ is given by $C(\Sigma)=\cup_{S \in \Sigma} C(S)$.

The axiom of choice is defined as follows.

Axiom of choice. Suppose that $\mathcal{T}$ is a collection of non-empty sets. Then there exists a function $\varphi: \mathcal{T} \rightarrow \cup_{T \in \mathcal{T}} T$ such that $\varphi(T) \in T$ for all $T \in \mathcal{T}$.

There are two basic forms of rationalizability properties that are commonly considered in the literature. The first is greatest-element rationalizability which requires the existence of a relation such that, for any feasible set, every chosen alternative is at least as good as every alternative in the set. Thus, this notion of rationalizability is based on the view that chosen alternatives should weakly dominate all feasible alternatives. Maximal-element rationalizability, on the other hand, demands the existence of a relation such that, for each feasible set, there exists no alternative in this set that is strictly preferred to any one of the chosen alternatives. Hence, this version of rationalizability does not require chosen alternatives to weakly dominate all elements of the feasible set but, instead, demands that they are not strictly dominated by any other feasible alternative.

In addition to one or the other of these two concepts of rationalizability, we have a choice regarding the properties that we require a rationalizing relation to possess. We consider the standard richness requirements of reflexivity and completeness and, in addition, the coherence properties of transitivity, quasi-transitivity, consistency and P-acyclicity. By combining each version of rationalizability with one or both (or none) of the richness conditions and with one (or none) of the coherence properties, various definitions of rationalizability are obtained. Some of these definitions are equivalent, others are independent, and some are implied by others. To get an understanding of what each of these definitions entails, we summarize all logical relationships between them in this section.

A choice function $C$ is greatest-element rationalizable, $G$-rationalizable for short, if there exists a relation $R$ on $X$, to be called a $G$-rationalization of $C$, such that $C(S)=G(S, R)$ for all $S \in \Sigma$. Analogously, a choice function $C$ is maximal-element rationalizable, M-rationalizable for short, if there exists a relation $R$ on $X$, to be called an $M$-rationalization of $C$, such that $C(S)=M(S, R)$ for all $S \in \Sigma$. If a rationalization $R$ is required to be reflexive and complete, the notion of greatestelement rationalizability coincides with that of maximal-element rationalizability because, in this case, $G(S, R)=M(S, R)$ for all $S \in \mathcal{X}$. Without these properties, however, this is not necessarily the case. Greatest-element rationalizability is based on the idea of chosen alternatives weakly dominating all alternatives in the feasible set under consideration, whereas maximal-element rationalizability requires chosen elements not to be strictly dominated by any other feasible 
alternative.

Depending on the additional properties that we might want to impose on a rationalization (if any), different notions of rationalizability can be defined. For simplicity of presentation, we use the following convention when formulating a rationalizability axiom. We distinguish three groups of properties of a relation, namely, rationalization properties, richness properties and coherence properties. The first group consists of the two rationalizability properties of Grationalizability and M-rationalizability, the second of the two requirements of reflexivity and completeness and, finally, the third of the axioms of transitivity, quasi-transitivity, consistency and P-acyclicity. Greatest-element rationalizability is abbreviated by $\mathbf{G}, \mathbf{M}$ is short for maximalelement rationalizability, $\mathbf{R}$ stands for reflexivity and $\mathbf{C}$ is completeness. Transitivity, quasitransitivity, consistency and P-acyclicity are denoted by $\mathbf{T}, \mathbf{Q}, \mathbf{S}$ and $\mathbf{A}$, respectively. We identify the property or properties to be satisfied within each of the three groups and separate the groups with hyphens. If none of the properties within a group is required, this is denoted by using the symbol $\emptyset$. Either greatest-element rationalizability or maximal-element rationalizability may be required. In addition to imposing one of the two richness properties only, reflexivity and completeness may be required simultaneously and we may require rationalizability properties without either of the two. We only consider notions of rationalizability involving at most one of the coherence properties at a time. As is the case for the richness properties, imposing none of the coherence properties is a possibility. Formally, a rationalizability property is identified by an expression of the form $\alpha-\beta-\gamma$, where $\alpha \in\{\mathbf{G}, \mathbf{M}\}, \beta \in\{\mathbf{R C}, \mathbf{R}, \mathbf{C}, \emptyset\}$ and $\gamma \in\{\mathbf{T}, \mathbf{Q}, \mathbf{S}, \mathbf{A}, \emptyset\}$. For example, greatest-element rationalizability by a reflexive, complete and transitive relation is denoted by G-RC-T, maximal-element rationalizability by a complete relation is $\mathbf{M}-\mathbf{C}-\emptyset$, greatest-element rationalizability by a reflexive and consistent relation is $\mathbf{G - R - S}$ and maximalelement rationalizability without any further properties of a rationalizing relation is $\mathbf{M}-\emptyset-\emptyset$. Clearly, according to this classification, there are $2 \cdot 4 \cdot 5=40$ versions of rationalizability.

In order to provide a benchmark for our subsequent analysis, we provide a full description of the logical relationships between these different notions of rationalizability. This result, which synthesizes the contributions of Bossert, Sprumont and Suzumura (2005a; 2005b; 2005c) can be found in Bossert and Suzumura (2005). For convenience, a diagrammatic representation is employed. All axioms that are depicted within the same box are equivalent, and an arrow pointing from one box $b$ to another box $b^{\prime}$ indicates that the axioms in $b$ imply those in $b^{\prime}$, and the converse implication is not true. In addition, of course, all implications resulting from chains of arrows depicted in the diagram are valid. 
Theorem 1 Suppose $C: \Sigma \rightarrow \mathcal{X}$ is a choice function with an arbitrary non-empty domain $\Sigma \subseteq$ $\mathcal{X}$. Then

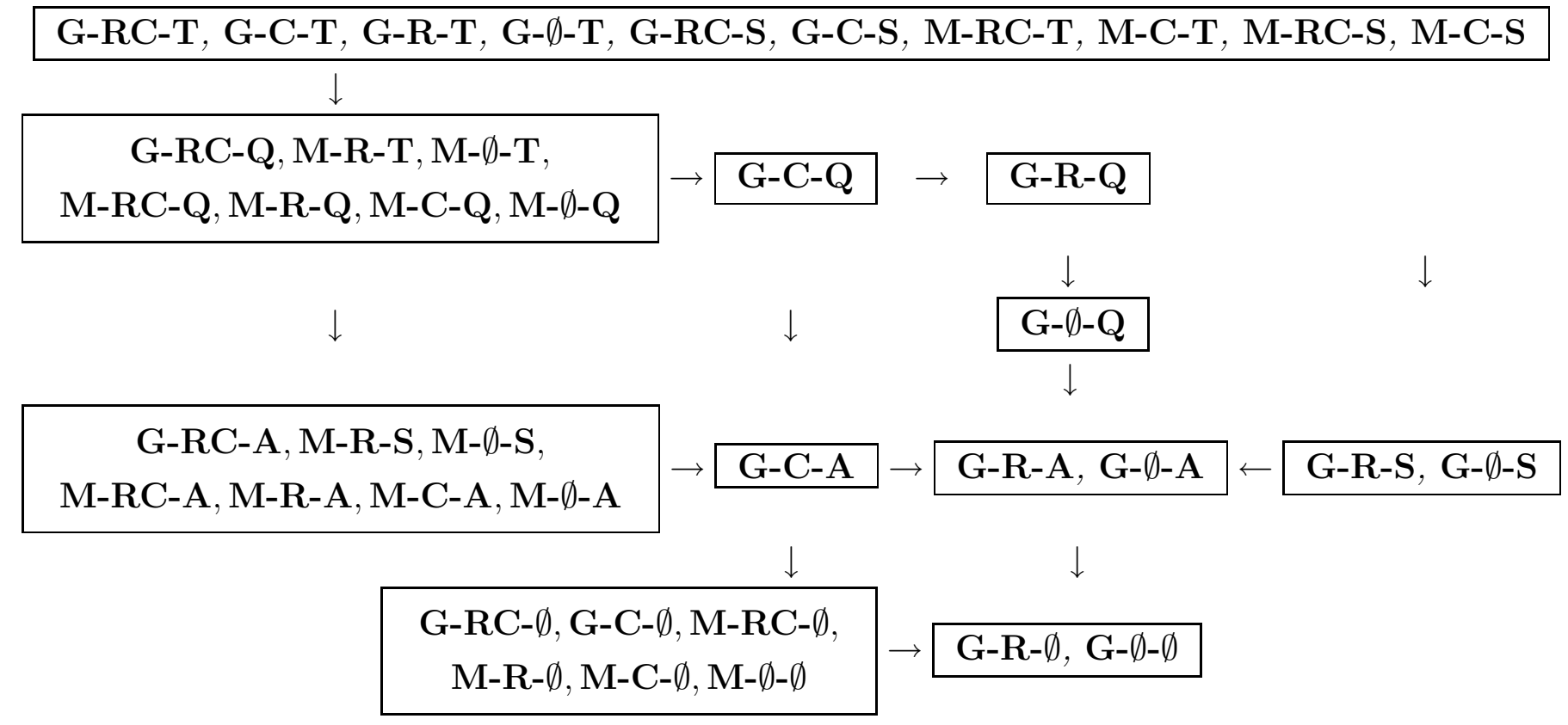

As is apparent from this theorem, M-rationalizability does not add any new versions of rationalizability, provided that all definitions of G-rationalizability with all of the four coherence properties are present.

\section{Domain Closedness Properties}

The theory of rationalizable choice functions on arbitrary domains is general enough to be applicable to whatever choice contexts we may care to specify. On the other hand, this generality is obtained at the cost of ignoring any structural conditions that may be imposed on the choice domain if we pay due attention to the specific choice context we are focusing on. An important class of domain restrictions that has been explored quite extensively in the literature on rational choice is based on set-theoretic closedness properties of the domain $\Sigma$. The two properties that have received the most attention are closedness under union and closedness under intersection. Closedness under union requires that, for any collection of sets in $\mathcal{X}$ each of which belongs to $\Sigma$, their union is a member of $\Sigma$ as well. Analogously, closedness under intersection demands that, whenever the members of a collection of sets in $\mathcal{X}$ belong to $\Sigma$ and their intersection is non-empty, then this intersection is an element of $\Sigma$ as well. 
These domain restrictions are of importance because they are plausible for several choice models. For example, closedness under intersection is satisfied by the domain consisting of all compact, convex and comprehensive subsets of $\mathbb{R}_{+}^{n}$, the non-negative orthant of Euclidean $n$ space for $n \in \mathbb{N}$. This domain is relevant in axiomatic models of bargaining where the elements of $\mathbb{R}_{+}^{n}$ are interpreted as possible utility or payoff distributions among $n \in \mathbb{N}$ agents that can be achieved through a bargaining process, and the origin represents the outcome that results if the agents fail to reach an agreement - the disagreement outcome. Recollect that feasible sets of utility distributions are usually assumed to be compact, convex and comprehensive.

Closedness under union is satisfied, for example, by the domain that consists of all compact and comprehensive (but not necessarily convex) subsets of $\mathbb{R}_{+}^{n}$ (or subsets of $\mathbb{R}^{n}$ with suitably modified definitions of comprehensiveness or a weakening of compactness to make the two properties compatible).

Up to now, the application of these types of domain restrictions has been limited to the study of full rationalizability - rationalizability by an ordering (recollect that in the case of an ordering, it is not necessary to specify whether greatest-element rationalizability or maximal-element rationalizability is considered because the two notions coincide). The usefulness of these domain assumptions lies in the fact that they allow us to work with properties that are weaker than others on arbitrary domains but turn out to be equivalent on suitably specified domains. In particular, the assumption of closedness under union implies that rationalizability by an ordering can be obtained as a consequence of a property that is, on arbitrary domains, considerably weaker than the requisite necessary and sufficient condition for that type of rationalizability. This property is Arrow's choice axiom; see Arrow (1959). Furthermore, while closedness under intersection is not sufficient to obtain the equivalence of Arrow's choice axiom and full rationalizability, it does imply that Arrow's choice axiom has some additional strength as compared to its power on arbitrary domains.

The purpose of this paper is to examine whether new logical relationships, in addition to those summarized in Theorem 1, emerge under one or the other of these closedness properties. It turns out that closedness under union leads to an additional implication, namely, that G-C-Q implies G-RC-A. It is worth mentioning that, unlike most of the earlier literature, we employ a closedness property with respect to set-theoretic unions that is not restricted to finite unions. The reason is that the argument employed to establish the above-mentioned new implication does not go through if attention is restricted to finite unions; this will become clear in the proof (which relies on the axiom of choice). In contrast, closedness under intersection does not give us any additional results: the same logical relationships as those of Theorem 1 are valid.

We now introduce the formal definitions of our closedness assumptions. A non-empty domain 
$\Sigma \subseteq \mathcal{X}$ is closed under union if and only if, for all non-empty collections $\mathcal{S}$ of elements of $\Sigma$, $\cup_{S \in \mathcal{S}} S \in \Sigma$. Analogously, $\Sigma$ is closed under intersection if and only if, for all non-empty collections $\mathcal{S}$ of elements of $\Sigma$, if $\cap_{S \in \mathcal{S}} S \neq \emptyset$, then $\cap_{S \in \mathcal{S}} S \in \Sigma$.

Requiring $\Sigma$ to be closed under union produces a new implication. In particular, it is now the case that G-C-Q implies G-RC-A so that we obtain the following theorem.

Theorem 2 Suppose $C: \Sigma \rightarrow \mathcal{X}$ is a choice function with a non-empty domain $\Sigma \subseteq \mathcal{X}$ which is closed under union. Then

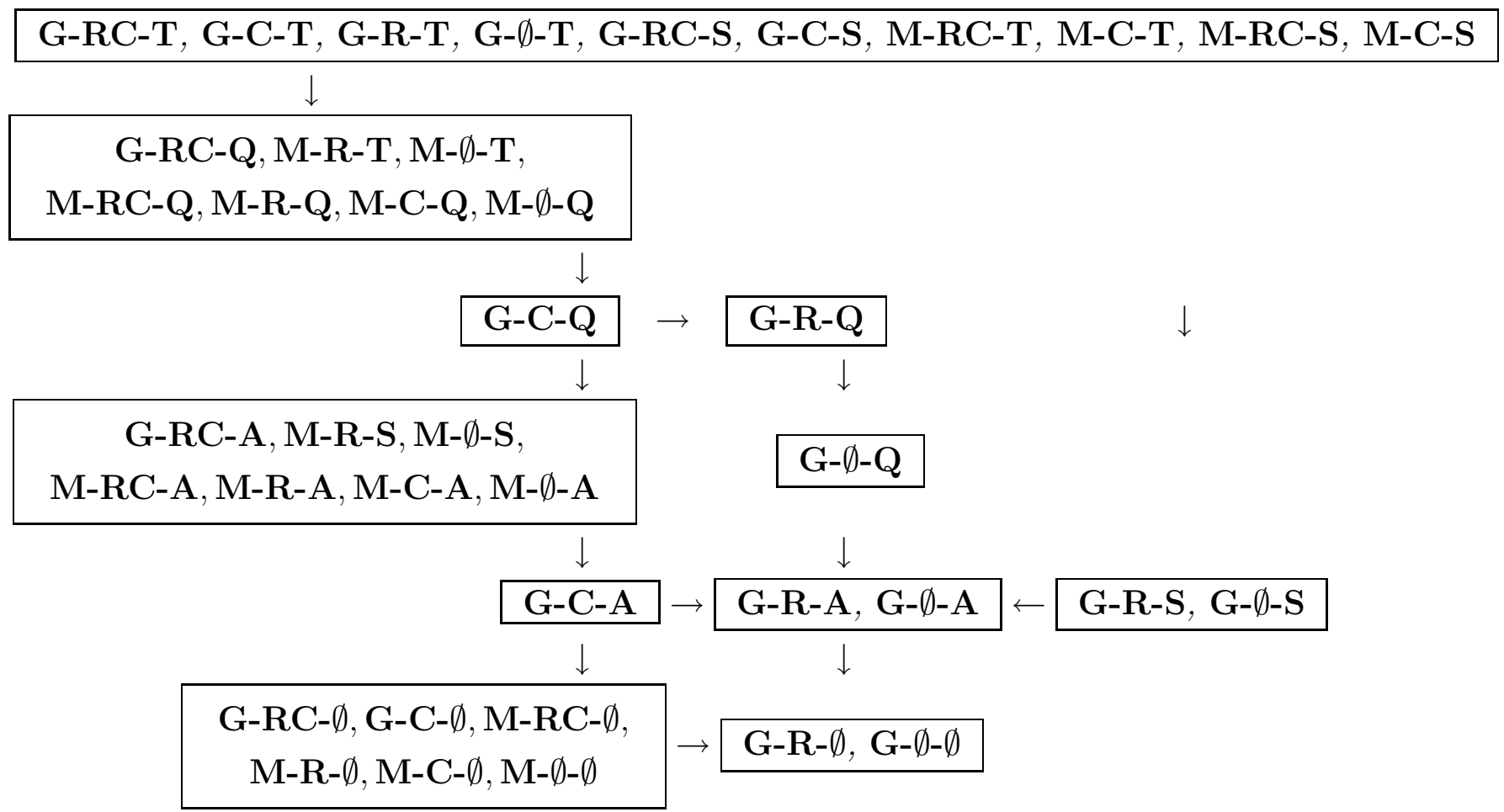

Proof. First, note that all the implications established in Theorem 1 remain valid because they apply to any domain (and, thus, in particular, to domains that are closed under union). Therefore, it remains to show that, provided that $\Sigma$ is closed under union, G-C-Q implies G-RC-A and that no implications other than those resulting from the theorem statement are valid.

(a) Suppose $\Sigma$ is closed under union, and let $R$ be a complete and quasi-transitive Grationalization of $C$. Define

$$
\mathcal{N}=\{(x, S) \mid(x, x) \notin R \text { and } S \in \Sigma \text { and }(x, y) \in R \text { for all } y \in S \backslash\{x\}\} .
$$

If $\mathcal{N}=\emptyset$, it follows immediately that $R \cup \Delta$ is a reflexive, complete and quasi-transitive (and thus P-acyclical) G-rationalization of $C$ and we are done. 
Now suppose $\mathcal{N} \neq \emptyset$. Let $\mathcal{Z}$ denote the set of all non-empty subsets of $X \times \Sigma$, and define a function $\tau: \Sigma \rightarrow \mathcal{Z}$ by $\tau(S)=\{(z, S) \mid z \in C(S)\}$ for all $S \in \Sigma$. Furthermore, let

$$
\mathcal{T}=\{\tau(S) \mid S \in \Sigma\}
$$

Let $\Phi$ be the set of all functions $\varphi: \mathcal{T} \rightarrow \cup_{T \in \mathcal{T}} T$ such that $\varphi(T) \in T$ for all $T \in \mathcal{T}$. By the axiom of choice, $\Phi \neq \emptyset$.

For all $\varphi \in \Phi$, define a function $g_{\varphi}: \mathcal{N} \rightarrow X$ by

$$
g_{\varphi}(x, S)=z \Leftrightarrow \varphi(\tau(S))=(z, S)
$$

for all $(x, S) \in \mathcal{N}$. Let

$$
\mathcal{G}=\left\{g: \mathcal{N} \rightarrow X \mid \text { there exists } \varphi \in \Phi \text { such that } g=g_{\varphi}\right\}
$$

The set $\mathcal{G}$ is non-empty because $\Phi$ is non-empty.

For all $g \in \mathcal{G}$, let

$$
R_{g}=R \cup \Delta \backslash\{(x, y) \mid \text { there exists } S \in \Sigma \text { such that }(x, S) \in \mathcal{N} \text { and } y=g(x, S)\} \text {. }
$$

Clearly, $R_{g}$ is reflexive because $\Delta \subseteq R$.

By the definitions of $\mathcal{N}$ and of $\mathcal{G},(x, y) \in I(R)$ for all pairs $(x, y)$ such that there exists $S \in \Sigma$ with $(x, S) \in \mathcal{N}$ and $y=g(x, S)$. Thus, $(x, y) \in P\left(R_{g}\right)$ for all such pairs which, together with the completeness of $R$, implies that $R_{g}$ is complete. This argument also establishes that $P(R) \subseteq P\left(R_{g}\right)$.

Next, we show that $R_{g}$ is a G-rationalization of $C$. Let $S \in \Sigma$ and $x \in S$.

Suppose first that $x \in C(S)$. Because $R$ is a G-rationalization of $C,(x, y) \in R$ for all $y \in S$ and in particular $(x, x) \in R$. By the definition of $\mathcal{N}$, this implies $(x, S) \notin \mathcal{N}$ which entails $(x, y) \in R_{g}$ for all $y \in S$. Thus, $x \in G\left(R_{g}, S\right)$.

Now suppose $x \in G\left(R_{g}, S\right)$. If there exists $y \in S \backslash\{x\}$ such that $(x, y) \notin R$, the completeness of $R$ implies that $(y, x) \in P(R) \subseteq P\left(R_{g}\right)$, contradicting the hypothesis $x \in G\left(R_{g}, S\right)$. If $(x, x) \notin R$, the definition of $R_{g}$ implies that $(x, g(x, S)) \notin R_{g}$, again contradicting the hypothesis $x \in$ $G\left(R_{g}, S\right)$ because, by definition, $g(x, S) \in S$. Thus, $(x, y) \in G(R, S)$ and $x \in C(S)$ follows from the assumption that $R$ G-rationalizes $C$.

We have now established that $R_{g}$ is a reflexive and complete G-rationalization of $C$ for all $g \in \mathcal{G}$. To complete the proof, we show that there must exist a $g \in \mathcal{G}$ such that $R_{g}$ is $\mathrm{P}$-acyclical.

A P-cycle in $R_{g}$ can be written as a pair $c=\left(K_{c},\left\{x_{c}^{0}, \ldots, x_{c}^{K_{c}}\right\}\right)$ where $K_{c} \in \mathbb{N} \backslash\{1\}$ and $x_{c}^{k} \in X$ for all $k \in\left\{0, \ldots, K_{c}\right\}$ such that $\left(x_{c}^{k-1}, x_{c}^{k}\right) \in P\left(R_{g}\right)$ for all $k \in\left\{1, \ldots, K_{c}\right\}$ and $\left(x_{c}^{K_{c}}, x_{c}^{0}\right) \in P\left(R_{g}\right)$. Without loss of generality, we can assume that the $x_{c}^{k}$ are pairwise distinct. 
Let $\mathcal{C}_{g}$ be the set of all P-cycles in $R_{g}$. We need to show that there exists $g \in \mathcal{G}$ such that $\mathcal{C}_{g}=\emptyset$. By way of contradiction, suppose that $\mathcal{C}_{g} \neq \emptyset$ for all $g \in \mathcal{G}$. Consider any $g \in \mathcal{G}$ and any $c \in \mathcal{C}_{g}$.

If there exists $k \in\left\{1, \ldots, K_{c}-1\right\}$ such that $\left(x_{c}^{k-1}, x_{c}^{k}\right) \in P(R)$ and $\left(x_{c}^{k}, x_{c}^{k+1}\right) \in P(R)$, the quasi-transitivity of $R$ implies $\left(x_{c}^{k-1}, x_{c}^{k+1}\right) \in P(R)$. The same reasoning implies that if $\left(x_{c}^{K_{c}-1}, x_{c}^{K_{c}}\right) \in P(R)$ and $\left(x_{c}^{K_{c}}, x_{c}^{0}\right) \in P(R)$, we must have $\left(x_{c}^{K_{c}-1}, x_{c}^{0}\right) \in P(R)$. Thus, because $K_{c}$ is finite, we can without loss of generality assume that, in any P-cycle, there are never two consecutive instances of strict preference according to $P(R)$.

If there exists $k \in\left\{1, \ldots, K_{c}-1\right\}$ such that $\left(x_{c}^{k-1}, x_{c}^{k}\right) \in P\left(R_{g}\right) \backslash P(R)$ and $\left(x_{c}^{k}, x_{c}^{k+1}\right) \in$ $P\left(R_{g}\right) \backslash P(R)$, the definition of $R_{g}$ implies that there exist $S, T \in \Sigma$ such that $\left(x_{c}^{k}, S\right) \in \mathcal{N}$, $x_{c}^{k-1}=g\left(x_{c}^{k}, S\right),\left(x_{c}^{k+1}, T\right) \in \mathcal{N}$ and $x_{c}^{k}=g\left(x_{c}^{k+1}, T\right)$. By definition of $\mathcal{N}$ and $g$, this implies $\left(x_{c}^{k}, x_{c}^{k}\right) \notin R$ and $x_{c}^{k} \in C(T)$, a contradiction to the assumption that $R$ is a G-rationalization of $C$. Thus, there cannot be two consecutive instances of strict preference according to $P\left(R_{g}\right) \backslash P(R)$ either.

Because $R$ is quasi-transitive and therefore $\mathrm{P}$-acyclical, every $\mathrm{P}$-cycle must contain at least one instance of strict preference according to $P\left(R_{g}\right) \backslash P(R)$. Because there cannot be any two consecutive instances of strict preference according to $P\left(R_{g}\right) \backslash P(R)$ and $K_{c} \geq 2$ for any P-cycle $c$ by assumption, it follows that every P-cycle must also contain at least one instance of strict preference according to $P(R)$.

Combining the findings of the previous three paragraphs, it follows that we can, without loss of generality, assume that all P-cycles alternate between instances of strict preference according to $P\left(R_{g}\right) \backslash P(R)$ and those according to $P(R)$. Thus, for all $g \in \mathcal{G}$ and for all P-cycles $c=$ $\left(K_{c},\left\{x_{c}^{0}, \ldots, x_{c}^{K_{c}}\right\}\right) \in \mathcal{C}_{g}$, we assume that $\left(x_{c}^{k-1}, x_{c}^{k}\right) \in P\left(R_{g}\right) \backslash P(R)$ for all odd $k \in\left\{1, \ldots, K_{c}\right\}$, $\left(x_{c}^{k-1}, x_{c}^{k}\right) \in P(R)$ for all even $k \in\left\{1, \ldots, K_{c}\right\}$, and $\left(x_{c}^{K_{c}}, x_{c}^{0}\right) \in P(R)$. Note that this implies that $K_{c}$ must be an odd number greater than or equal to three. Furthermore, for all odd $k \in\left\{1, \ldots, K_{c}\right\}$, there exists $S_{c}^{k} \in \Sigma$ such that $\left(x_{c}^{k}, S_{c}^{k}\right) \in \mathcal{N}$ and $x_{c}^{k-1}=g\left(x_{c}^{k}, S_{c}^{k}\right)$.

Let $\Upsilon$ be the set of all alternatives in $C(\Sigma)$ that do not appear in any P-cycle. That is, $x \in C(\Sigma)$ is an element of $\Upsilon$ if and only if, for all $g \in \mathcal{G}$, for all $c \in \mathcal{C}_{g}$ and for all odd $k \in\left\{1, \ldots, K_{c}\right\}, x_{c}^{k-1} \neq x$. Now define $\Sigma^{\prime}=\{S \in \Sigma \mid C(S) \cap \Upsilon=\emptyset\}$, that is, $\Sigma^{\prime}$ is composed of those sets $S$ in $\Sigma$ such that all elements of $C(S)$ appear in some P-cycle. Note that $\Sigma^{\prime}=\Sigma$ is possible. Let, for all sets $S \in \Sigma \backslash \Sigma^{\prime}, y_{S}$ be an alternative in $C(S)$ that does not appear in any P-cycle. Let $\mathcal{G}^{\prime}$ be the subset of $\mathcal{G}$ containing all functions $g$ such that, for all $(x, S) \in \mathcal{N}$ with $S \in \Sigma \backslash \Sigma^{\prime}, g(x, S)=y_{S}$. Clearly, $\mathcal{G} \neq \emptyset$. If $\Sigma^{\prime}=\emptyset$, any $g \in \mathcal{G}^{\prime}$ is such that $\mathcal{C}_{g}=\emptyset$, a 
contradiction. Therefore, $\Sigma^{\prime} \neq \emptyset$. Let

$$
T=\bigcup_{g \in \mathcal{G}^{\prime}} \bigcup_{c \in \mathcal{C}_{g}} \bigcup_{k \in\left\{1, \ldots, K_{c}\right\}} S_{c}^{k} .
$$

Because $\mathcal{G}^{\prime}$ is non-empty and $\mathcal{C}_{g}$ is non-empty for all $g \in \mathcal{G}^{\prime}, T \neq \emptyset$. Because $\Sigma$ is closed under union, $T \in \Sigma$. By definition, all sets $S_{c}^{k}$ in (2) are elements of $\Sigma^{\prime}$. Because $C$ is G-rationalizable by $R$, it follows that, for all $y \in S_{c}^{k} \backslash C\left(S_{c}^{k}\right)$, there exists $x \in S_{c}^{k} \subseteq T$ such that $(y, x) \notin R$. Again invoking the G-rationalizability of $C$ by $R$, this implies that

$$
C(T)=\bigcup_{g \in \mathcal{G}^{\prime}} \bigcup_{c \in \mathcal{C}_{g}} \bigcup_{k \in\left\{1, \ldots, K_{c}\right\}} C\left(S_{c}^{k}\right) .
$$

Consider any $g \in \mathcal{G}^{\prime}, c \in \mathcal{C}_{g}$ and $k \in\left\{1, \ldots, K_{c}\right\}$, and let $x \in C\left(S_{c}^{k}\right)$. Because $S_{c}^{k} \in \Sigma^{\prime}$, there exists a P-cycle containing $x$. Thus, there exists $g^{\prime} \in \mathcal{G}^{\prime}, c^{\prime} \in \mathcal{C}_{g}$ and $y \in X$ such that $(x, y) \in P\left(R_{g}\right) \backslash P(R)$. Furthermore, there exist $z, w \in X$ such that $(z, w) \in P\left(R_{g}\right) \backslash P(R)$ and $(w, x) \in P(R)$. By definition, there exists $S \in \Sigma^{\prime}$ such that $w \in S \subseteq T$ and, because $R$ is a G-rationalization of $C, x \notin C(T)$. This argument applies to all elements in the union on the right side of (3) and, therefore, $C(T)=\emptyset$, a contradiction.

To prove that no further implications other than those resulting from the arrows depicted in the theorem statement are valid, it is sufficient to provide examples showing that (b) G-RC-Q does not imply G- $\emptyset$-S; (c) G- $\emptyset$-S does not imply G- $\emptyset$-Q; (d) G- $\emptyset$-S does not imply G-C- $\emptyset$; (e) G-RC-A does not imply G- $\emptyset$-Q; (f) G-C-A does not imply G-RC-A; (g) G-R-Q does not imply G-C- $\emptyset$; (h) G- $\emptyset$-Q does not imply G-R-Q; and (i) G-C- $\emptyset$ does not imply G- $\emptyset$-A. All of these examples are such that $\Sigma$ is closed under union and under intersection.

(b) To show that G-RC-Q does not imply G- $(\emptyset-\mathbf{S}$, consider the following example. Let $X=$ $\{x, y, z\}$ and $\Sigma=\mathcal{X}$. Define the choice function $C$ by letting $C(\{x\})=\{x\}, C(\{x, y\})=\{x\}$, $C(X)=\{x, z\}, C(\{x, z\})=\{x, z\}, C(\{y\})=\{y\}, C(\{y, z\})=\{y, z\}$ and $C(\{z\})=\{z\}$. This choice function is G-rationalizable by the reflexive, complete and quasi-transitive relation

$$
R=\{(x, x),(x, y),(x, z),(y, y),(y, z),(z, x),(z, y),(z, z)\} .
$$

Suppose $R^{\prime}$ is a G-rationalization of $C$. Because $y \in C(\{y, z\})$, we have $(y, y) \in R^{\prime}$. Therefore, $y \notin C(\{x, y\})$ implies $(x, y) \in P\left(R^{\prime}\right)$. Because $y \in C(\{y, z\})$, we have $(y, z) \in R^{\prime}$ and, analogously, because $z \in C(\{x, z\})$, we have $(z, x) \in R^{\prime}$. This implies that $R^{\prime}$ cannot be consistent.

(c) We now prove that G- $\emptyset$-S does not imply G- $\emptyset$-Q. Let $X=\{x, y, z, w\}$ and $\Sigma=$ $\{X,\{x, y, w\},\{y\},\{y, z\},\{z\}\}$. Define the choice function $C$ by letting $C(X)=\{w\}, C(\{x, y, w\})=$ $\{x, w\}, C(\{y\})=\{y\}, C(\{y, z\})=\{y\}$ and $C(\{z\})=\{z\}$. This choice function is G-rationalizable 
by the consistent relation

$$
R=\{(x, x),(x, y),(x, w),(y, y),(y, z),(z, z),(w, x),(w, y),(w, z),(w, w)\}
$$

Suppose $C$ is G-rationalizable by a quasi-transitive relation $R^{\prime}$. Because $y \in C(\{y, z\})$, we have $(y, y) \in R^{\prime}$. Therefore, $y \notin C(\{x, y\})$ implies $(x, y) \in P\left(R^{\prime}\right)$. Analogously, $z \in C(\{z\})$ implies $(z, z) \in R^{\prime}$ and, therefore, $z \notin C(\{y, z\})$ implies $(y, z) \in P\left(R^{\prime}\right)$. Because $R^{\prime}$ is quasi-transitive, it follows that $(x, z) \in P\left(R^{\prime}\right)$ and hence $(x, z) \in R^{\prime}$. Furthermore, because $R^{\prime}$ is a G-rationalization of $C$ and $x \in C(\{x, y, w\})$, we must have $(x, x) \in R^{\prime},(x, y) \in R^{\prime}$ and $(x, w) \in R^{\prime}$. Thus, $x \in G\left(X, R^{\prime}\right)$ and, because $R^{\prime}$ is a G-rationalization of $C$, it follows that $x \in C(X)$, contradicting the definition of $C$.

(d) Next, we prove that G- $\emptyset$-S does not imply G-C- $\emptyset$. Let $X=\{x, y, z\}$ and $\Sigma=$ $\{\{x\},\{x, y\}, X,\{x, z\}\}$. Define the choice function $C$ by letting $C(\{x\})=\{x\}, C(\{x, y\})=$ $\{x, y\}, C(X)=\{x\}$ and $C(\{x, z\})=\{x, z\} . C$ is G-rationalizable by the consistent relation

$$
R=\{(x, x),(x, y),(x, z),(y, x),(y, y),(z, x),(z, z)\}
$$

but it does not have a complete G-rationalization. By way of contradiction, suppose $R^{\prime}$ is such a relation. By completeness, we must have $(y, z) \in R^{\prime}$ or $(z, y) \in R^{\prime}$. Suppose first that $(y, z) \in R^{\prime}$. Because $R^{\prime}$ is a G-rationalization of $C$ and $y \in C(\{x, y\})$, it follows that $(y, x) \in R^{\prime}$ and $(y, y) \in R^{\prime}$. Together with $(y, z) \in R^{\prime}$ and the assumption that $R^{\prime}$ is a G-rationalization of $C$, we obtain $y \in C(X)$, contradicting the definition of $C$. Now suppose $(z, y) \in R^{\prime}$. Because $R^{\prime}$ is a G-rationalization of $C$ and $z \in C(\{x, z\})$, it follows that $(z, x) \in R^{\prime}$ and $(z, z) \in R^{\prime}$. Together with $(z, y) \in R^{\prime}$ and the assumption that $R^{\prime}$ is a G-rationalization of $C$, we obtain $z \in C(X)$, again contradicting the definition of $C$.

(e) To prove that $\mathbf{G - R C}-\mathbf{A}$ does not imply $\mathbf{G}-\emptyset$-Q , let $X=\{x, y, z\}$ and $\Sigma=\mathcal{X}$. Define the choice function $C$ by $C(\{x\})=\{x\}, C(\{x, y\})=\{x\}, C(X)=\{x\}, C(\{x, z\})=\{x, z\}$, $C(\{y\})=\{y\}, C(\{y, z\})=\{y\}$ and $C(\{z\})=\{z\} . C$ is G-rationalizable by the reflexive, complete and P-acyclical relation

$$
R=\{(x, x),(x, y),(x, z),(y, y),(y, z),(z, x),(z, z)\}
$$

By way of contradiction, suppose $R^{\prime}$ is a quasi-transitive G-rationalization of $C$. Because $y \in$ $C(\{y, z\}), z \in C(\{z\})$ and $z \notin C(\{y, z\})$, the assumption that $R^{\prime}$ is a G-rationalization of $C$ implies $(y, z) \in P\left(R^{\prime}\right)$. Furthermore, because $x \in C(\{x, y\})$ and $y \notin C(\{x, y\})$, we obtain $(x, y) \in P\left(R^{\prime}\right)$. Because $R^{\prime}$ is quasi-transitive, it follows that $(x, z) \in P\left(R^{\prime}\right)$. This, in turn, implies $(z, x) \notin R^{\prime}$ and, because $R^{\prime}$ is a G-rationalization of $C, z \notin C(\{x, z\})$, contradicting the definition of $C$. 
(f) To show that G-C-A is not sufficient to imply G-RC-A, let $X=\{x, y, z, w\}$ and $\Sigma=$ $\{X,\{x, y, w\},\{x, w\},\{y\},\{y, z\},\{y, z, w\},\{y, w\},\{w\}\}$. Define a choice function $C$ by letting $C(X)=\{w\}, C(\{x, y, w\})=\{x, w\}, C(\{x, w\})=\{x, w\}, C(\{y\})=\{y\}, C(\{y, z\})=\{y\}$, $C(\{y, z, w\})=\{y, w\}, C(\{y, w\})=\{y, w\}$ and $C(\{w\})=\{w\}$. This choice function is Grationalizable by the complete and $\mathrm{P}$-acyclical relation

$$
R=\{(x, x),(x, y),(x, w),(y, y),(y, z),(y, w),(z, x),(z, y),(w, x),(w, y),(w, z),(w, w)\} .
$$

Suppose $R^{\prime}$ is a reflexive, complete and P-acyclical G-rationalization of $C$. Reflexivity implies that we must have $(z, z) \in R^{\prime}$. This, in turn, implies $(y, z) \in P\left(R^{\prime}\right)$ because $C(\{y, z\})=\{y\}$. Furthermore, because $C(\{y, w\})=\{y, w\}$, we must have $(y, y) \in R^{\prime}$ and $(y, w) \in R^{\prime}$. Thus, because $y \notin C(\{x, y, w\})$, it must be the case that $(y, x) \notin R^{\prime}$ and, because $R^{\prime}$ is complete, $(x, y) \in P\left(R^{\prime}\right)$. Because $x \in C(\{x, y, w\})$, it follows that $(x, x) \in R^{\prime},(x, y) \in R^{\prime}$ and $(x, w) \in R^{\prime}$. Thus, $x \notin C(X)$ implies $(x, z) \notin R^{\prime}$. The completeness of $R^{\prime}$ implies $(z, x) \in P\left(R^{\prime}\right)$, contradicting the P-acyclicity of $R^{\prime}$.

(g) To see that G-R-Q does not imply G-C- $\emptyset$, consider the following example. Let $X=$ $\{x, y, z\}, \Sigma=\{\{x\},\{x, y\}, X,\{x, z\}\}, C(\{x\})=\{x\}, C(\{x, y\})=\{x, y\}, C(X)=\{x\}$ and $C(\{x, z\})=\{x, z\} . C$ is G-rationalizable by the reflexive and quasi-transitive relation

$$
R=\{(x, x),(x, y),(x, z),(y, x),(y, y),(z, x),(z, z)\},
$$

but it does not have a complete G-rationalization. By way of contradiction, suppose $R^{\prime}$ is a complete G-rationalization of $C$. Completeness implies that we must have $(y, z) \in R^{\prime}$ or $(z, y) \in R^{\prime}$. Suppose first that $(y, z) \in R^{\prime}$ is true. Because $R^{\prime}$ is a G-rationalization of $C$ and $y \in C(\{x, y\})$, it follows that $(y, x) \in R^{\prime}$ and $(y, y) \in R^{\prime}$. Together with $(y, z) \in R^{\prime}$ and the definition of G-rationalizability, we obtain $y \in C(X)$, contradicting the definition of $C$. Now suppose $(z, y) \in R^{\prime}$. Because $R^{\prime}$ is a G-rationalization of $C$ and $z \in C(\{x, z\})$, it follows that $(z, x) \in R^{\prime}$ and $(z, z) \in R^{\prime}$. Together with $(z, y) \in R^{\prime}$ and the definition of G-rationalizability, we obtain $z \in C(X)$, again contradicting the definition of $C$.

(h) To show that $\mathbf{G}-\emptyset-\mathbf{Q}$ does not imply G-R-Q, consider the following example. Let $X=$ $\{x, y, z, w\}$ and $\Sigma=\{\{x, y\}, X,\{x, y, w\},\{y\},\{y, z, w\},\{y, w\}\}$, and define the choice function $C$ by letting $C(\{x, y\})=\{y\}, C(X)=\{w\}, C(\{x, y, w\})=\{y, w\}, C(\{y\})=\{y\}, C(\{y, z, w\})=$ $\{z, w\}$ and $C(\{y, w\})=\{y, w\}$. This choice function is G-rationalizable by the quasi-transitive relation

$$
R=\{(x, y),(x, w),(y, x),(y, y),(y, w),(z, y),(z, z),(z, w),(w, x),(w, y),(w, z),(w, w)\} .
$$

Suppose $R^{\prime}$ is a reflexive and quasi-transitive G-rationalization of $C$. By reflexivity, $(x, x) \in R^{\prime}$ and, because $x \notin C(\{x, y\})$ and $y \in C(\{x, y\})$, we must have $(y, x) \in P\left(R^{\prime}\right)$ and $(y, y) \in R^{\prime}$. 
Because $y \in C(\{x, y, w\})$, we have $(y, w) \in R^{\prime}$. Hence, $y \notin C(\{y, z, w\})$ implies $(y, z) \notin R^{\prime}$ because $R^{\prime}$ is a G-rationalization of $C$. Because $z \in C(\{y, z, w\})$, the assumption that $R^{\prime}$ is a G-rationalization of $C$ implies $(z, y) \in R^{\prime}$ and, thus, $(z, y) \in P\left(R^{\prime}\right) . R^{\prime}$ being quasi-transitive, we obtain $(z, x) \in P\left(R^{\prime}\right)$. Because $z \in C(\{y, z, w\})$, it follows that $(z, y) \in R^{\prime},(z, z) \in R^{\prime}$ and $(z, w) \in R^{\prime}$. Together with $(z, x) \in P\left(R^{\prime}\right) \subseteq R^{\prime}$ and the assumption that $R^{\prime}$ is a G-rationalization of $C$, we obtain $z \in C(X)$, which contradicts the definition of $C$.

(i) We now show that G-C- $\emptyset$ does not imply G- $\emptyset$-A. Let $X=\{x, y, z, w\}$ and $\Sigma=$ $\{X,\{x, y, w\},\{x, z, w\},\{x, w\},\{y, z, w\},\{y, w\},\{z, w\},\{w\}\}$. Define the choice function $C$ by letting $C(X)=\{w\}, C(\{x, y, w\})=\{x, w\}, C(\{x, z, w\})=\{z, w\}, C(\{x, w\})=\{x, w\}$, $C(\{y, z, w\})=\{y, w\}, C(\{y, w\})=\{y, w\}, C(\{z, w\})=\{z, w\}$ and $C(\{w\})=\{w\} . C$ is G-rationalizable by the complete relation $R$ given by

$$
\{(x, x),(x, y),(x, w),(y, y),(y, z),(y, w),(z, x),(z, z),(z, w),(w, x),(w, y),(w, z),(w, w)\}
$$

but it does not have a P-acyclical G-rationalization. Suppose $R^{\prime}$ is a G-rationalization of $C$. Because $C(\Sigma)=X$ and $R^{\prime}$ G-rationalizes $C$, it follows that $\Delta \subseteq R^{\prime}$. Because $y \notin C(\{x, y, w\})$ and $y \in C(\{y, z, w\})$, the assumption that $R^{\prime}$ is a G-rationalization of $C$ implies that we must have $(x, y) \in P\left(R^{\prime}\right)$. Analogously, $[x \notin C(\{x, z, w\})$ and $x \in C(\{x, y, w\})]$ implies $(z, x) \in P\left(R^{\prime}\right)$, and $[z \notin C(\{y, z, w\})$ and $z \in C(\{x, z, w\})]$ implies $(y, z) \in P\left(R^{\prime}\right)$. Therefore, $R^{\prime}$ cannot be Pacyclical.

Closedness under intersection does not change the results reported in Theorem 1. To see this, note first that all the implications established in that theorem remain true because they are valid on any domain (and, thus, in particular, on domains that are closed under intersection). Furthermore, the examples employed in the proof of Theorem 1 are all defined on domains that are closed under intersection and, in addition, G-C-Q does not imply G-RC-A even if $\Sigma$ is closed under intersection. To see that this is the case, consider the following example, which establishes the claim. Let $X=\{x, y, z, w\}$ and $\Sigma=\{\{x, y, w\},\{x, w\},\{y\},\{y, z\},\{y, z, w\},\{y, w\},\{w\}\}$, and define $C(\{x, y, w\})=\{w\}, C(\{x, w\})=\{w\}, C(\{y\})=\{y\}, C(\{y, z\})=\{y\}, C(\{y, z, w\})=$ $\{y\}, C(\{y, w\})=\{y, w\}$ and $C(\{w\})=\{w\}$. This choice function is G-rationalizable by the complete and quasi-transitive relation

$$
R=\{(x, y),(x, z),(x, w),(y, y),(y, z),(y, w),(z, x),(z, y),(z, w),(w, x),(w, y),(w, w)\} .
$$

Suppose $R^{\prime}$ is a reflexive, complete and P-acyclical G-rationalization of $C$. Because $C(\{y, z\})=$ $\{y\}$ and $R^{\prime}$ is reflexive, we obtain $(y, z) \in P\left(R^{\prime}\right)$. Analogously, because $C(\{x, w\})=\{w\}$ and $R^{\prime}$ is reflexive, we must have $(w, x) \in P\left(R^{\prime}\right)$. Because $y \in C(\{y, w\})$ and $y \notin C(\{x, y, w\})$, we must 
have $(y, x) \notin R^{\prime}$ and, because $R^{\prime}$ is complete, it follows that $(x, y) \in P\left(R^{\prime}\right)$. Analogously, because $w \in C(\{y, w\})$ and $w \notin C(\{y, z, w\})$, we must have $(w, z) \notin R^{\prime}$ and, because $R^{\prime}$ is complete, it follows that $(z, w) \in P\left(R^{\prime}\right)$. Therefore, we have established that $(x, y) \in P\left(R^{\prime}\right),(y, z) \in P\left(R^{\prime}\right)$, $(z, w) \in P\left(R^{\prime}\right)$ and $(w, x) \in P\left(R^{\prime}\right)$, contradicting the P-acyclicity of $R^{\prime}$.

\section{Concluding Remarks}

Domain closedness properties are not the only domain assumptions that are of interest in the analysis of rational choice. For example, binary domains are considered in Bossert, Sprumont and Suzumura (2005a) and base domains are analyzed in Bossert, Sprumont and Suzumura (2005c). A domain is a binary domain if it contains all two-element subsets of the universal set, and a base domain contains all subsets of the universal set of cardinality one and two. As established in the above-mentioned contributions, these domain assumptions lead to dramatic simplifications of the characterization results that can be obtained for various notions of rationality. Further domains, such as those consisting of sets that are bounded by hyperplanes, remain to be explored further in future work.

\section{References}

Arrow, K.J. (1959): "Rational Choice Functions and Orderings," Economica, Vol.26, pp.121127.

Bossert, W. (1999): "Intersection Quasi-Orderings: An Alternative Proof," Order, Vol.16, pp.221-225.

Bossert, W., Y. Sprumont and K. Suzumura (2005a): "Consistent Rationalizability," Economica, Vol.72, pp.185-200.

Bossert, W., Y. Sprumont and K. Suzumura (2005b): "Maximal-Element Rationalizability," Theory and Decision, Vol.58, pp.325-350.

Bossert, W., Y. Sprumont and K. Suzumura (2005c): "Rationalizability of Choice Functions on General Domains Without Full Transitivity," forthcoming in Social Choice and Welfare.

Bossert, W. and K. Suzumura (2005): "Rational Choice on General Domains," unpublished manuscript, CIREQ, Université de Montréal. 
Donaldson, D. and J.A. Weymark (1998): "A Quasiordering is the Intersection of Orderings," Journal of Economic Theory, Vol.78, pp.382-387.

Dushnik, B. and E.W. Miller (1941): "Partially Ordered Sets," American Journal of Mathematics, Vol.63, pp.600-610.

Luce, R.D. (1956): "Semiorders and the Theory of Utility Discrimination," Econometrica, Vol.24, pp.178-191.

Richter, M.K. (1966): "Revealed Preference Theory," Econometrica, Vol.41, pp.1075-1091.

Richter, M.K. (1971): "Rational Choice," in J.S. Chipman, L. Hurwicz, M.K. Richter and H.F. Sonnenschein, eds., Preferences, Utility, and Demand, New York: Harcourt Brace Jovanovich, pp.29-58.

Sen, A.K. (1969): "Quasi-Transitivity, Rational Choice and Collective Decisions," Review of Economic Studies, Vol.36, pp.381-393.

Sen, A.K. (1970): Collective Choice and Social Welfare, San Francisco: Holden-Day.

Suzumura, K. (1976): "Remarks on the Theory of Collective Choice," Economica, Vol.43, pp.381-390.

Suzumura, K. (1983): Rational Choice, Collective Decisions and Social Welfare, New York: Cambridge University Press.

Szpilrajn, E. (1930): "Sur l'Extension de l'Ordre Partiel," Fundamenta Mathematicae, Vol.16, pp.386-389. 